\title{
Real Time Text Mining on Twitter Data
}

\author{
Shilpy Gandharv \\ M. Tech (CSE) \\ LNCT, Bhopal M.P., India
}

\author{
Vivek Richhariya \\ Prof. Dept. of CSE \\ LNCT, Bhopal M.P., India
}

\author{
Vineet Richhariya, PhD \\ Prof. \& Head, Dept. of CSE \\ LNCT, Bhopal M.P., India
}

\begin{abstract}
Social media constitute a challenging new source of information for intelligence gathering and decision making. Twitter is one of the most popular social media sites and often becomes the primary source of information. Twitter messages are short and well suited for knowledge discovery. Twitter provides both researchers and practitioners a free Application Programming Interface (API) which allows them to gather and analyse large data sets of tweets. Twitter information aren't solely tweet texts, as Twitter's API provides a lot of info to perform attention-grabbing analysis studies. The paper concisely describes method of knowledge gathering and therefore the main areas of knowledge mining, information discovery and information visual image from Twitter information. In this paper we can create a twitter app from which we can fetch the real time twitter tweets on a particular topic and stored it into R and then we can apply several text mining steps on the tweets to pre-process the tweets text and than we can analyse the preprocess data by visualizing them.
\end{abstract}

\section{Keywords}

Twitter data, text mining, real time, visualization, NLP, wordcloud, ggplot2

\section{INTRODUCTION}

Over past ten years, industries and organizations doesn't have demand to store and perform operations and analytics on info of the consumers. However around from 2005, the requirement to rework everything into information is way amused to satisfy the wants of the individuals. Therefore huge information came into image within the real time business analysis of process information. From twentieth century ahead this World Wide Web has modified the means of expressing their views. gift scenario is totally they're expressing their thoughts through online blogs, discussion forms and additionally some on-line applications like Facebook, Twitter, etc [3]. If we have a tendency to take Twitter as our example nearly 1TB of text information is generating inside per week within the sort of tweets. So, by this it's perceive clearly however this web is ever-changing the means of living and elegance of individuals. Among these tweets will be classified by the hash worth tags that they're commenting and posting their tweets. So, currently several corporations and additionally the survey corporations area unit mistreatment this for performing some analytics[5] specified they will predict the success rate of their product or additionally they will show the various read from the information that they need collected for analysis. But, to calculate their views is extremely troublesome during a traditional means by taking these serious information that area unit about to generate day by day.

Text mining has become a preferred approach to analysing and understanding massive datasets not done by the traditional analysis techniques. These tools are applied to a spread of data issues, like understanding themes in social media or facilitating info retrieval in unstructured information. Text mining is also a extraordinarily great tool at intervals the beginnings of research exploration, allowing the matter info to counsel themes and concepts to the scientist throughout analysis. this may provides a useful begin line for framing further analysis queries and analysis approaches, notably if hypotheses Associate in Nursing any queries don't seem to be proverbial (as is typical with an inductive analysis approach). Moreover, these tools can also assist in improvement and structuring text-based info for future analysis in representation or completely different graphical tools. And, in addition to the tangible analysis benefits, text mining is also a fun and fruitful technique of discovery! Text Mining [4], is one of the foremost frequent however tough exercise faced by beginners in IP / analytics consultants. the most important challenge is one has to completely assess the underlying patterns in text, that too manually. For example: it's pretty common to delete numbers from the text before we have a tendency to do any reasonably text mining. However what if we would like to extract one thing like " $24 / 7$ ". Hence, the text cleansing exercise is very customized as per the target of the exercise and therefore the kind of text patterns.

$\mathbf{R}$

$\mathrm{R}$ [9] is each a language and surroundings orienting towards applied math computing and graphics creation (R Core Team, 2016). $\mathrm{R}$ is created on the market below the antelope General Public License; as results of sturdy community involvement, there are various extensions, referred to as packages, developed over time, likewise as sturdy documentation. For this extensibility and flexibility, $\mathrm{R}$ has remained consistently common for information and text mining applications across many domains, and includes powerful text mining tools.

Here, we'll specialize in $\mathrm{R}$ packages helpful in understanding and extracting insights from the text and text mining packages.

In this paper, we are going to be following subsequent packages:

1. tm, framework for text mining applications

2. SnowballC, text stemming library

3. ggplot2, one of the best data visualization libraries

4. Wordcloud, for making wordcloud visualizations

\section{LITERATURE REVIEW}

According to [1], Text mining, conjointly noted as text data processing, is that the method of extracting attention-grabbing and non-trivial patterns or data from text documents. It uses algorithms to rework free flow text (unstructured) into knowledge which will be analysed (structured) by applying applied mathematics, Machine Learning and language process (NLP) techniques. Text mining is associate degree evolving technology that enables enterprises to know their customers 
well, and facilitate them in redefining client desires. As ecommerce is changing into additional and skilful, the quantity of client reviews and feedback that a product receives has big quickly over a amount of your time. For a popular asset, the number of review comments can be in thousands or even more. This makes it difficult for the manufacturer to read all of them to make an informed decision in improving product quality and support. Again it is difficult for the manufacturer to keep track and to manage all customer opinions. This article attempts to derive some meaningful information from asset reviews which will be used in enhancing asset features from engineering point of view and helps in improving the support quality and customer experience.

In [8] ,they present a system for the acquisition, analysis and visualisation of Twitter data. Twitter messages are harvested and keep in a very distributed cluster, and also the knowledge is processed victimization algorithms enforced in a very MapReduce framework. we tend to gift a clump rule capable of characteristic the most topics of interest in a very tweet knowledge set. Also, we tend to design a visualisation technique that permits to follow the intensity of twitter activity at a given geographical location. during this paper we've got bestowed a system for the acquisition, analysis and visualization of Twitter knowledge. Twitter messages are harvested and keep in a very distributed cluster, and he knowledge is processed victimization algorithms enforced in a very MapReduce framework. we tend to bestowed a clump rule capable of characteristic hot topics of interest in a very tweet knowledge set. Also, we tend to designed a visualisation technique that permits to follow the density of twitter activity in a very given geographical location. The system could be a model and was meant to gift the potential use of a social media platform as supply of enormous scale spatio-temporal data. It represents the building ground for future social media connected applications targeting a mess of doable applications with high social impact like emergency state of affairs management, risk and harm assessment and even social unrest.

In this paper they can visualize the twitter data using matlab and matlab is a traditional technique which can not handle bigdata, And twitter data generates huge amount of data per data which is not able to process by traditional tools and technique, due to which we need a powerful visualizing techniques which can work on bigdata directly.

In [2],Twitter, as a social media could be a very fashionable manner of expressing opinions and interacting with others within the on-line world. Once taken in aggregation tweets will give a mirrored image of public sentiment towards events. During this paper, we offer a positive or negative sentiment on Twitter [12] posts employing a well-known machine learning methodology for text categorization. Additionally, we tend to use manually labeled (positive/negative) tweets to make a trained methodology to accomplish a task. The task is probing for a correlation between twitter sentiment and events that have occurred. The trained model relies on the Bayesian supply Regression (BLR) classification methodology. We tend to used external lexicons to notice subjective or objective tweets, other Unigram and written word options and used TF-IDF (Term Frequency-Inverse Document Frequency) to strain the options. Exploitation the FIFA journey 2014 as our case study, we tend to used Twitter Streaming API and a few of the official tourney hashtags to mine, filter and method tweets, so as to research the reflection of public sentiment towards sudden events. An equivalent approach will be used as a basis for predicting future events. Twitter, one in all the foremost common on-line social media and micro-blogging services, could be a very fashionable methodology for expressing opinions and interacting with others within the on-line world Twitter messages give real data within the format of short texts that categorical opinions, ideas and events captured within the moment. Tweets (Twitter posts) are well-suited sources of streaming information for opinion mining and sentiment polarity detection Opinions, evaluations, emotions and speculations usually mirror the states of individuals; they contains narrow-minded information expressed during a language composed of subjective expressions .During this paper, we tend to examine the effectiveness of a usually used text categorization methodology known as Bayesian supply Regression (BLR) Classification for providing positive or negative sentiment on tweets. We tend to use extracted Twitter sentiment to seem for correlations between this sentiment and major FIFA journey 2014 events as our case study.

In this paper the author calculate the polarity of the tweets with the help of Bayesian supply Regression (BLR) classification methodology and predict some events based on correlation between the events. But this methodology fails when data is very huge in terms of pettabyte and also it cannot do real time analysis, for this we need a new tool and technique which can handle such huge and large datasets.

\section{PROBLEM DEFINITION}

Text mining [7] facilitate a company derive doubtless valuable business insights from text-based content like word documents, email and postings on social media streams like Facebook, Twitter and LinkedIn. data processing or Text mining plays a vital role in deciding as a result of through these mining techniques we will analyse the info and on the idea of result we will take a call. Now a days social media sites like twitter are widely used to share user opinions on various topics, twitter gives a platform to user to share their views and thoughts on various field like political, industrial, education and there is a petabytes of data generated by twitter in a day.

So the mining techniques are used to analysis the social twitter data thorough we get large amount of datasets to analysis, so the analysis of twitter data provides a better way for making decision.

\section{MOTIVATION}

Text mining [11] is not yet a part of mainstream predictive analytics, though it is on the short list for many organizations. But text mining is difficult, requiring additional expertise and processing complementary to predictive modelling, but not often taught in machine learning or statistics computing.

Nevertheless, text mining is being used increasingly as organizations recognize the untapped information contained in text. Social media, such as Twitter [13] and Facebook, have been used effectively by organizations to uncover trends that, when identified through text mining, can be used to leverage the positive trends.

\section{OBJECTIVE}

Data preprocessing could be a data processing technique that involves reworking information into a visible format. Realworld information is commonly incomplete, inconsistent, and/or lacking in sure behaviours or trends, and is probably going to contain several errors. Information preprocessing could be a well-tried methodology of resolution such problems. Information preprocessing prepares information for any process. 
Preprocessing steps contains many objective. they're as follows:

1.Fetch information from Twitter Streaming API.

2.Convert unstructured JSON information into structured information.

3.Apply stemming to get rid of stop words.

4.Store the preprocessed information for analysis.

\section{PROPOSED WORK}

The work involved in this usually requires several computational techniques (such as data and text mining, natural language processing, etc.) and complex analytical processes required to manipulate varied data sources. Besides that, reach a point of balance between the computational side of the process and the aesthetic side using tables, charts, colors and other visual features, could favor a good analysis and quicker understanding of such data. In a process to derive the target outcome from the unstructured raw text which we fetching from web, the first step is to identify suitable data source.

Pre-processing method plays a very important role in text mining techniques and applications. It is the first step in the text mining process. In this dissertation, we discuss the three packages comes in $\mathrm{R}$ language through which we can perform the text mining on twitter data. Text mining of Twitter data with $\mathrm{R}$ packages twitter, tm and ggplot2

\section{PROPOSED METHODOLOGY:}

Our Steps or Algorithm Steps will follow:

1. First we get a complex social data and stored .

2. when retrieving we have a tendency to reworked the text, tweets ar 1st reborn to an information frame then to a corpus. After that, the corpus desires a handful of transformations, as well as dynamical letters to small letter, removing punctuations/numbers and removing stop words.

3. In several cases, words got to be stemmed to retrieve their radicals. as an example, "example" and "examples" area unit each stemmed to "exampl". However, after that, one may want to complete the stems [6] to their original forms, so that the words would look "normal".

4. After transforming and stemming process is done then we build a document term matrix. Based on the matrix, many data mining tasks can be done, for example, clustering, classification and association analysis.

5. With the help of matrix we can identify the frequent words and their association between words.

6. After building a document-term matrix, we can now visualize the outputs.

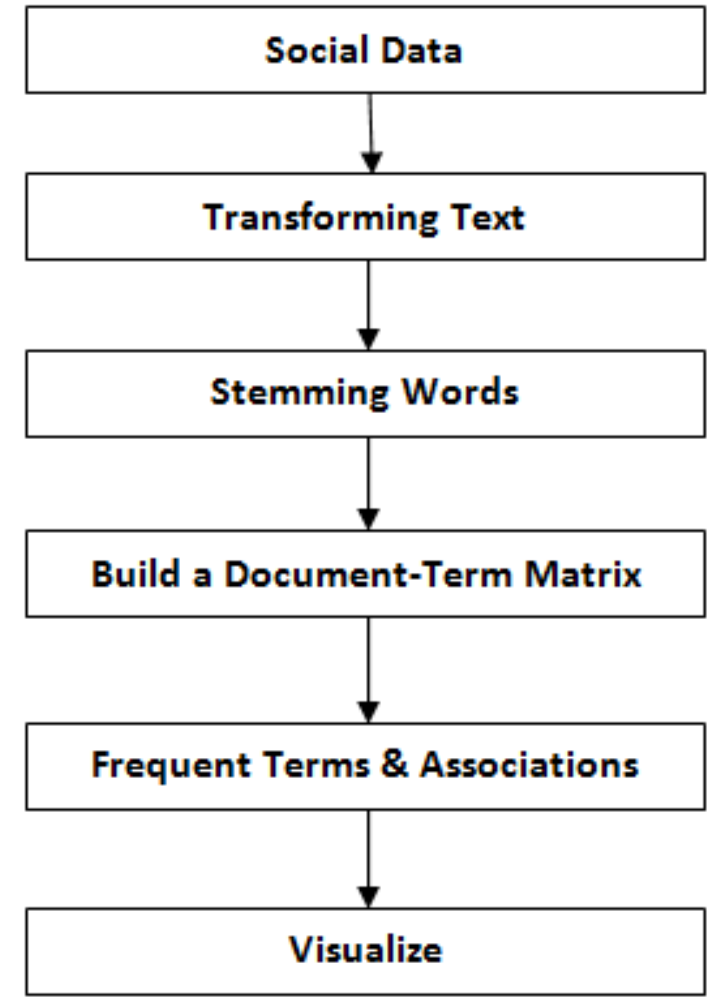

\section{Proposed Flow Diagram}

\section{EXPERIMENTAL \& RESULT ANALYSIS}

All the experiments were performed using an i5-2410M CPU @ $2.30 \mathrm{GHz}$ processor and $4 \mathrm{~GB}$ of RAM running Windows. After that we can install $r$ base core on windows and Rstudio [10] and than we are fetching real tweets and after that performing text mining on that collected tweets. So, to achieve this we are going to follow the following methods:

$>$ Gathering the tweets

$>$ Perform text mining on Tweets.

$>$ Analyze the pre-process data.

\subsection{Gathering the tweets}

Any social media investigation is only as good as the data used for its analysis. The process of social media analysis involves essentially four steps: data identification, data analysis, data interpretation and, finally, information presentation. The main problem is how to extract the information that is available on Twitter and how it can be used to draw meaningful insight. To achieve this, first there is a need to build a data analyser for tweets. Tweets are available to researchers and practitioners through public Twitter APIs. Twitter allows developers to collect data via Twitter REST API (https://dev.twitter.com/rest/public/) and the Streaming API (https://dev.twitter.com/streaming/overview).

First of all if we want to do analysis on Twitter data we want to get Twitter data first so to get it we want to create an account in Twitter developer and create an application by clicking on the new application button provided by them shown in figure 1 , After creating a new application just create the access tokens so that we need to provide our authentication details there and also after creating application it will be having one consumer keys to access that application for getting Twitter data. 


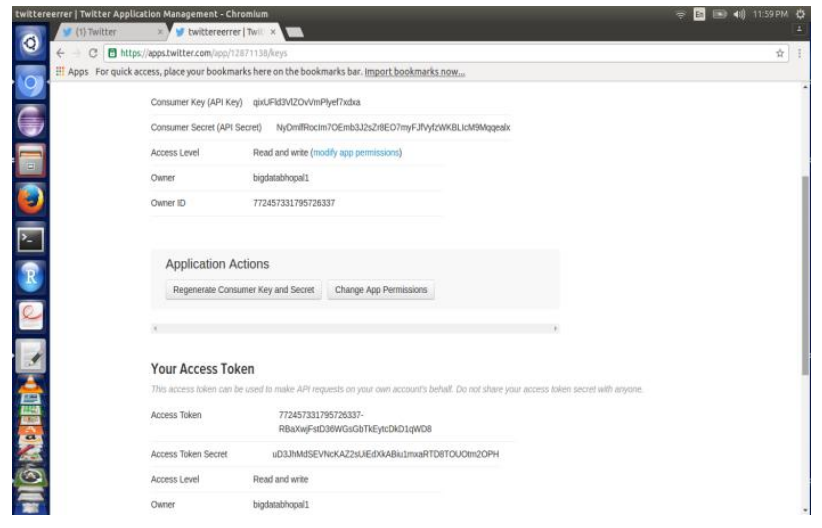

Figure 1. Generating access keys from twitter application

\subsection{Perform text mining on tweets}

For performing text mining on tweets we need a package called tm package which internally consist natural language processing NLP package. Figure 2 shows the loading and performing operation through tm package.

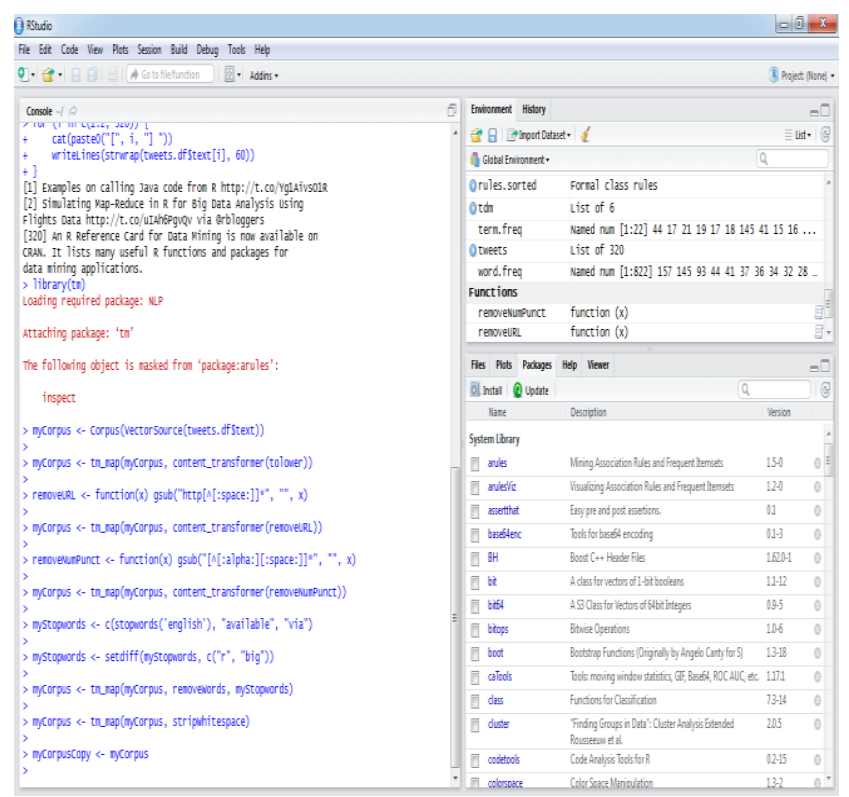

Figure 2 performing text preprocessing using tm package

We are using corpus for preprocessing twitter text and tm package consist many function through which we can remove numbers, punctual, and special characters coming from text. And first we are converting all the text into lower case to remove noise and we can also eliminate url's coming from text.

After preprocessing using tm package we can perfrom stemming on preprocessed data for this we can use SnowballC package. Figure 3 describe the stemming is perform on preprocesssed text. For this we can collects same meaning types word separately such as example, exampl, examples comes into same category.

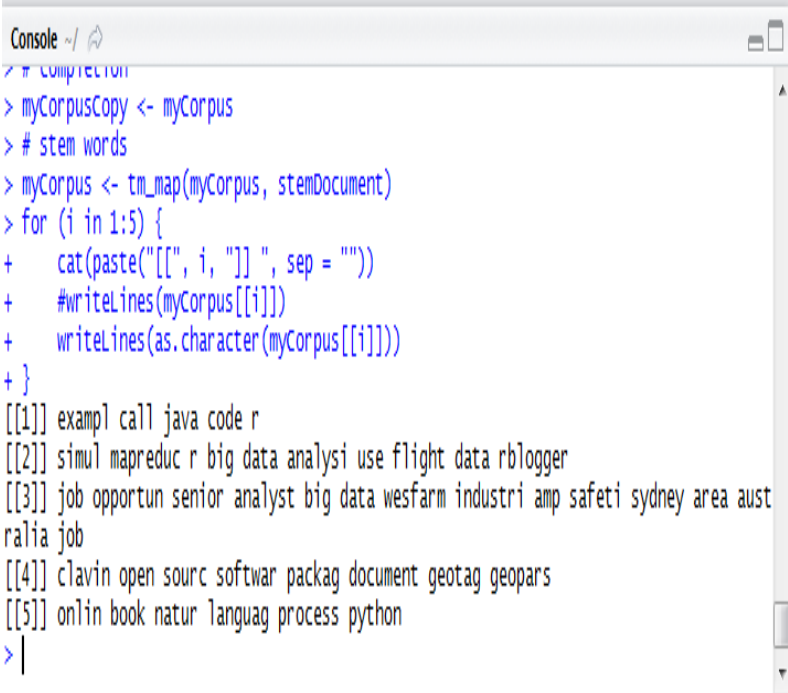

Figure 3. Applying stemming on preprocessed text

After stemming process we can developed a term-document matrix and find the frequency of each term using association properties. Figure 4 describe the operation on tdm (term document matrix) and finding most frequent keywords .

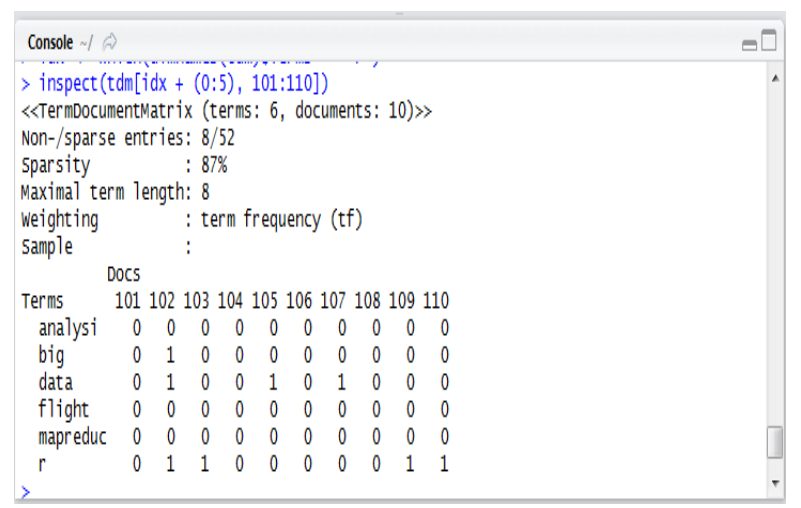

Figure 4. Term-Document matrix

After creating document term matrix we can find the frequent terms and find those terms who are having maximum frequency. Figure 5 shows the frequent terms.

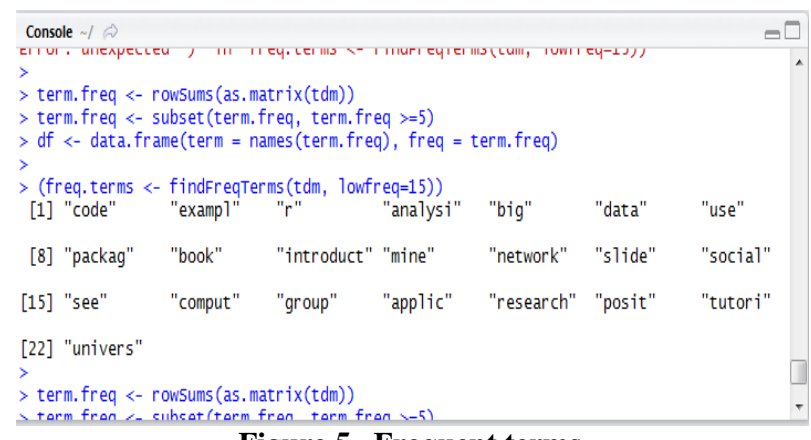

Figure 5 . Frequent terms

\subsection{Analyze the pre-process data}

After text mining using tm package we can analyse the text mining result using visualizing package called ggplot 2 , In tm package we can find the frenquent terms and using ggplot 2 we can visualize these frequent terms. Figure 6 shows the ggplot for frequent terms along according to their count. 


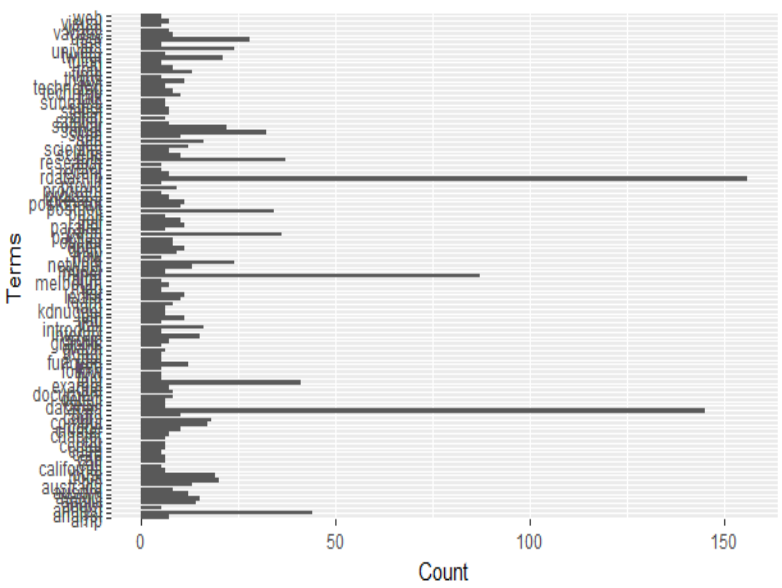

Figure 6. ggplot for frequent terms

After plotting the frequent term using ggplot2 we are now plots the word cloud for frequent terms which comes from the various text preprocessing steps. A word cloud is a simple yet informative way to understand textual data and to do text analysis. Figure 7 shows the word cloud of frequent terms.

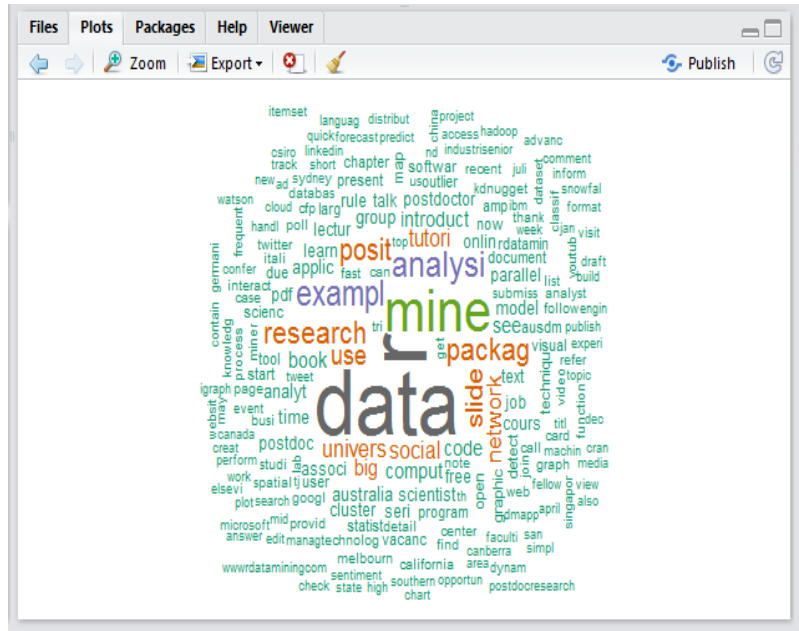

Figure 8. WordCloud for frequent terms

\section{CONCLUSION}

Twitter data is very useful in decision making because its provide a variety of opinions on various topics. so the texting mining will perform on twitter data and we are using a visualizing techniques. In this paper we can create a twitter app from which we can fetch the real time twitter tweets on a particular topic and stored it into $\mathrm{R}$ and than we can apply several text mining steps on the tweets to pre-process the tweets text and then we can analyse the preprocess data by visualizing them.

\section{REFERENCES}

[1] Chandrasekhar Rangu, Shuvojit Chatterjee, Srinivasa Rao Valluru, "Text Mining Approach for Product Quality Enhancement" in IEEE 2017.

[2] Mr. Peiman Barnaghi and John G. Breslin , “, Opinion Mining and sentiment polarity on Twitter and correlation between Events \& Sentiment", International Conference on Big Data Computing and Application , IEEE 2016.

[3] Judith Sherin Tilsha S, Shobha M.S.," A Survey on Twitter Data Analysis Techniques to Extract Public Opinion.”, IJARCSE , Vol. 5, Issue 11, Nov 2015, 2277128X

[4] Lokmanyathilak Govindan Sankar Selvan," A Framework for Fast-Feedback Opinion Mining on Twitter Data Streams", IEEE 2015.

[5] T. K. Das , D.P. Acharjya \& M. R. Patra, " Opinion Mining about a product by Analyzing Public Tweets in Twitter “, ICCCI- 2014, Jan 03-05, 2014.

[6] Porter M.F, Snowball: A language for stemming algorithms. 2001.

[7] Ning Zhong, Yuefeng $\mathrm{Li}$, and Sheng-Tang $\mathrm{Wu}$, "Effective Pattern Discovery for Text Mining", IEEE Transactions on Knowledge And Data Engineering, Vol. 24, No.1, January 2012.

[8] Andrei Sechelea, Tien Do Huu, Evangelos Zimos, and Nikos Deligiannis, "Twitter Data Clustering and Visualization", in 2016 23rd International Conference on Telecommunications (ICT), 2016 IEEE.

[9] Shruti Kohli, Himani Singal, "Data Analysis with R" in 2014 IEEE/ACM 7th International Conference on Utility and Cloud Computing.

[10] Arun Jalanila, Nirmal Subramanian, "Comparing SAS® Text Miner, Python, R" in 2016 IEEE International Conference on Healthcare Informatics.

[11] Dr. S. Vijayarani, Ms. J. Ilamathi, Ms. Nithya, “ Preprocessing Techniques for Text Mining - An Overview" in International Journal of Computer Science \& Communication Networks, Vol 5(1),7-16.

[12] Pearanalitycs, "Twitter study — august 2009," 2009 [Online]. Available: http://pearanalytics.com/wpcontent/uploads/2009/08/ Twitter-Study-August-2009.pdf

[13] S. Kumar, F. Morstatter, and H. Liu, Twitter Data Analytics. $\quad$ Springer, $\quad$ Aug. 2013. 